\title{
IMPACT OF SPATIAL CHANNEL CORRELATION ON SUPER QUASI-ORTHOGONAL SPACE-TIME TRELLIS CODES
}

\author{
Biljana Badic, Alexander Linduska, Hans Weinrichter \\ Institute for Communications and Radio Frequency Engineering \\ Vienna University of Technology, \\ Gusshausstr.25, A-1040 Vienna, Austria \\ Email: bbadic@nt.tuwien.ac.at
}

\begin{abstract}
The error performance of a new family of super quasi- orthogonal space-time trellis codes (S-QSTTCs) for four transmit antennas on correlated MIMO channels is investigated. Two slightly different designs of S-QSTTCs, namely S-QSTTCs obtained by concatenating the extended Alamouti space-time code (EAC) with a simple outer trellis code and the concatenation of the ABBA code with a simple outer trellis code are compared in detail. It is shown that EAtype and ABBA-type S-QSTTCs perform quite different on spatially correlated MIMO channels. However, a unique labelling of the trellis diagram adapted to these two super quasi-orthogonal constituent code types, results in a very similar error performance even in spatially correlated channels.
\end{abstract}

\section{INTRODUCTION}

Space-time coding is a promising technique to improve the reliability of multiple-input/multiple-output (MIMO) systems. Two different space-time coding methods, namely space-time trellis coding (STTC) and space-time block coding (STBC) have been proposed. STTC has been introduced in [1] as a coding technique that promises full diversity and high coding gain at the price of a quite high decoding complexity. An STBC that promises full diversity and full data rate for two transmit antennas was introduced by Alamouti [2]. For more than two transmit antennas many different designs have been proposed providing either full diversity [3], or full data rate [4], [5].

In [6] it has been shown that the concatenation of a STBC with an outer trellis code results in a powerful code with reduced data rate, since the number of available transmit matrices in every state of the trellis is smaller than the maximum number of matrices the STBC could offer. Parameterized STBCs which provide additional transmit matrices have been introduced in [7] and [8] to increase the data rate. These parameterized STBCs are called super orthogonal STBCs (S-OSTBCs) in case of 2 transmit antennas. In case of 4 transmit antennas a set of super quasi-orthogonal STBCs (S-QSTBCs) can be obtained by parametrization of a quasiorthogonal space-time block code (QSTBC). In connection with an outer-trellis code specific subsets of these S-QSTBCs are used as constituent codes which are assigned to the various trellis branches. Every constituent code can be characterized by specified values of the parameters used to generate the S-QSTBC.

The combination of several constituent orthogonal spacetime block codes with an outer trellis code is called a super orthogonal space-time trellis code (S-OSTTC) and the combination of several constituent quasi-orthogonal space-time block codes with an outer trellis code is called a super quasiorthogonal space-time trellis code (S-QSTTC). Every edge of the trellis is assigned a complete constituent code, such that the resulting code provides high diversity and coding gain at full data rate.

In this paper, several S-QSTTCs over spatially correlated MIMO channels are investigated. We study two slightly different S-QSTTCs using either the ABBA code [4] or the extended Alamouti code (EAC) [5] as the basic QSTBC to build the constituent codes for the resulting S-QSTTC. In Section II we give an overview of an S-OSTTC and present two design examples of S-QSTTCs. In Section III we discuss the performance of S-QSTBCs. Section IV analyzes the performance of the S-QSTTC on correlated channels. Section $\mathrm{V}$ concludes the paper.

\section{SUPER QUASI-ORTHOGONAL SPACE-TIME TRELLIS CODES (S-QSTTC)}

\subsection{Super-Orthogonal Space-Time Trellis Codes for Two Transmit Antennas}

In this subsection, we discuss the combination of a simple inner STBC with an outer trellis code [7] to obtain a powerful trellis code with full diversity and substantial coding 
gain at full data rate. We start with the well-known Alamouti STBC [2] with the transmit matrix:

$$
\mathbf{C}=\left(\begin{array}{rr}
s_{1} & s_{2} \\
-s_{2}^{*} & s_{1}^{*}
\end{array}\right) .
$$

By multiplying of the first column of $\mathbf{C}$ with $e^{j \theta}$, a parameterized O-STBC is obtained:

$$
\mathbf{C}^{\theta}=\left(\begin{array}{rr}
s_{1} e^{j \theta} & s_{2} \\
-s_{2}^{*} e^{j \theta} & s_{1}^{*}
\end{array}\right) .
$$

$\theta$ is restricted to values that do not expand the original signal constellation. This means, for an L-PSK signal constellation, $\theta_{l}=2 \pi l / L$ with $l=0,1, \cdots, L-1$. For BPSK $\theta_{l} \in\{0, \pi\}$, for QPSK and QAM $\theta_{l} \in\{0, \pi / 2, \pi, 3 \pi / 2\}$. Such symbol rotations do not change the diversity order and orthogonality of $\mathbf{C}$, since $\mathbf{C}^{\theta} \mathbf{C}^{\theta H}=\mathbf{C}^{\theta H} \mathbf{C}^{\theta}=\mathrm{s}^{2} \mathbf{I}$, with $\mathrm{s}^{2}=\left|s_{1}\right|^{2}+\left|s_{2}\right|^{2}$ and $\mathbf{I}$ being the $2 \times 2$ identity matrix. A code $\mathbf{C}^{\theta}$ with a specific value of $\theta$ is used as a constituent code in super orthogonal space-time trellis codes for two transmit antennas [7]. This coding strategy assures, that the resulting S-OSTTC achieves the same diversity as the original STBC at full data rate. The complexity of the Viterbi decoder at the receiver is rather low due to the orthogonality of the constituent STBCs.

\subsection{Super-Quasi-Orthogonal Space-Time Trellis Code for Four Transmit Antennas}

Orthogonal space-time block codes providing full data rate do not exist for more than two transmit antennas. Lower rate OSTBCs for more than two transmit antennas have been proposed in [4], [5]. In [8] S-QSTTC have been introduced as an extension of S-OSTTC for two transmit antennas to four transmit antennas. These S-QSTBCs can be used to obtain a new family of trellis codes which provide the diversity of the original QSTBC at full data rate and provide additional coding gain [8].

In the following we explain the design of a S-QSTTC in detail. In the first design step we select an appropriate QSTBC. In fact, we focus on the EAC [5]

$$
\mathbf{C}_{E A}=\left(\begin{array}{rrrr}
s_{1} & s_{2} & s_{3} & s_{4} \\
-s_{2}^{*} & s_{1}^{*} & -s_{4}^{*} & s_{3}^{*} \\
-s_{3}^{*} & -s_{4}^{*} & s_{1}^{*} & s_{2}^{*} \\
s_{4} & -s_{3} & -s_{2} & s_{1}
\end{array}\right)
$$

and on the ABBA code [4]

$$
\mathbf{C}_{A B B A}=\left(\begin{array}{rrrr}
s_{1} & s_{2} & s_{3} & s_{4} \\
-s_{2}^{*} & s_{1}^{*} & -s_{4}^{*} & s_{3}^{*} \\
s_{3} & s_{4} & s_{1} & s_{2} \\
-s_{4}^{*} & s_{3}^{*} & -s_{2}^{*} & s_{1}^{*}
\end{array}\right)
$$

Using quasi-orthogonal design, pairs of transmitted symbols can be decoded independently and the loss of diversity in
QSTBC is due to some coupling term between the estimated symbols. The QSTBCs in (3) and (4) show the same performance on identically distributed (i.i.d.) channels, but it has been shown in [4] that the ABBA code is very sensitive against channel correlation what leads to collapsing all decoding algorithms.

As in the case of two transmit antennas, additional transmit matrices are needed to obtain a full rate STTC. By multiplying the first column of $\mathbf{C}$ by $e^{j \Theta_{1}}$ and the second column by $e^{j \Theta_{2}}$ a super set of quasi-orthogonal codes is obtained with

$$
\mathbf{C}_{E A}^{\Theta_{1}, \Theta_{2}}=\left(\begin{array}{rrrr}
s_{1} e^{j \Theta_{1}} & s_{2} e^{j \Theta_{2}} & s_{3} & s_{4} \\
-s_{2}^{*} e^{j \Theta_{1}} & s_{1}^{*} e^{j \Theta_{2}} & -s_{4}^{*} & s_{3}^{*} \\
-s_{3}^{*} e^{j \Theta_{1}} & -s_{4}^{*} e^{j \Theta_{2}} & s_{1}^{*} & s_{2}^{*} \\
s_{4} e^{j \Theta_{1}} & -s_{3} e^{j \Theta_{2}} & -s_{2} & s_{1}
\end{array}\right)
$$

if $\mathbf{C}_{E A}$ is used, and

$$
\mathbf{C}_{A B B A}^{\Theta_{1}, \Theta_{2}}=\left(\begin{array}{rrrr}
s_{1} e^{j \Theta_{1}} & s_{2} e^{j \Theta_{2}} & s_{3} & s_{4} \\
-s_{2}^{*} e^{j \Theta_{1}} & s_{1}^{*} e^{j \Theta_{2}} & -s_{4}^{*} & s_{3}^{*} \\
s_{3} e^{j \Theta_{1}} & s_{4} e^{j \Theta_{2}} & s_{1} & s_{2} \\
-s_{4}^{*} e^{j \Theta_{1}} & s_{3}^{*} e^{j \Theta_{2}} & -s_{2}^{*} & s_{1}^{*}
\end{array}\right)
$$

if $\mathbf{C}_{A B B A}$ is used. Equations (5) and (6) provide super sets of quasi-orthogonal space-time block codes with two parameters $\Theta_{1}$ and $\Theta_{2}$ that provide enough transmit matrices to design a full-rate S-QSTTC. $\mathbf{C}_{E A}^{\Theta_{1}, \Theta_{2}}$ is called an EA super-set of quasi- orthogonal codes and $\mathbf{C}_{A B B A}^{\Theta_{1}, \Theta_{2}}$ is called an ABBA super-set of quasi-orthogonal codes. As in the case of SOSTTC, $\Theta_{1}$ and $\Theta_{2}$ are constrained such that the original signal constellation is preserved. Again QSTBCs with specified values of $\Theta_{1}$ and $\Theta_{2}$ are used as constituent codes of the S-QSTTC.

The set of all constituent codes derived from a basic QSTBC (EA or ABBA) with constrained parameters $\Theta_{1}$ and $\Theta_{2}$ is denoted as super quasi-orthogonal (SQO) code set. For example, the SQO code set for the EA STBC applying BPSK modulation is denoted as $\tilde{\mathbf{C}}_{E A}=\left\{\mathbf{C}_{E A}^{0,0}, \mathbf{C}_{E A}^{0, \pi}, \mathbf{C}_{E A}^{\pi, 0}, \mathbf{C}_{E A}^{\pi, \pi}\right\}$. To design powerful S-QSTTCs an appropriate Set-Partitioning of the constituent codes has to be performed. This means, that the set of all matrices within a constituent STBC has to be iteratively partitioned into smaller sub-sets with increasing minimum distance between the matrices within a sub-set. A detailed description of the principles of set-partitioning can be found in [7]. The appropriate distance metric used in this partitioning is the determinant of the distance matrix A of two code matrices. In this paper the sub-sets of the first partitioning step are denoted as $S_{0}, S_{1}$. Trellis-coding with the super quasi-orthogonal code set is performed in such a way, that each trellis-state is assigned a complete constituent code and every edge diverging from this state is assigned a complete sub-set $S_{0}$ or $S_{1}$ obtained from this constituent code. This coding strategy assures, that the re- 
sulting S-QSTTC achieves the same diversity as the original QSTBC and achieves full data rate. Decoding with the Viterbi algorithm is quite easy due to the quasi-orthogonality of the constituent STBCs.

Fig. 1 shows an example of a 2-state S-QSTTC. The la-

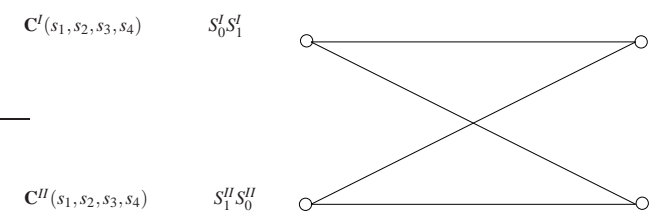

Figure 1: 2-state S-QSTTC transmitting QPSK symbols.

belling in Fig. 1 indicates that in the upper state of the trellis the subset $S_{0}^{I}$ of the constituent QSTBC $\mathbf{C}_{I}$ is assigned to the upper edge diverging from this state whereas the subset $S_{1}^{I}$ from $\mathbf{C}_{I}$ is assigned to the lower edge diverging from the upper state. With this trellis, we generate three different $\mathrm{S}$ QSTTCs by choosing different rotation parameters $\Theta_{1}$ and $\Theta_{2}$ using the EAC STBC (5) and another three different SQSTTC by using the ABBA STBC (6).

In Tab. 1 the constituent codes $\mathbf{C}^{I}$ and $\mathbf{C}^{I I}$ for the three $\mathbf{S}$ QSTTC code examples are listed. Such constituents codes can be derived either from the basic EA-STBC or from the ABBA STBC.

\begin{tabular}{|c|c|c|}
\hline & $\mathbf{C}^{\prime}$ & $\mathbf{C}^{\prime \prime}$ \\
\hline Trellis-Code 1 & $\mathbf{C}^{0,0}$ & $\mathbf{C}^{0, \pi}$ \\
\hline Trellis-Code 2 & $\mathbf{C}^{\pi, 0}$ & $\mathbf{C}^{0, \pi}$ \\
\hline Trellis-Code 3 & $\mathbf{C}^{0,0}$ & $\mathbf{C}^{\pi, \pi}$ \\
\hline
\end{tabular}

Table 1: Constituent Codes for the Trellis-example in Fig.1.

\subsection{Transmission Scheme}

The complete transmission scheme with $n_{t}=4$ transmit antennas and $n_{r}=1$ receive antenna can be described by $\mathbf{y}=$ $\sqrt{E_{b}} \mathbf{D h}+\mathbf{n}$, where $\mathbf{y}$ is the $\left(N_{l} \times 1\right)$ vector of signal samples received at the single receive antenna within $N_{l}$ successive time slots, with $N_{l}$ denoting the frame length. $E_{b}$ is the mean bit energy per transmit antenna, $\mathbf{D}$ is a $N_{l} \times n_{t}$ transmitted codeword matrix with $D_{k i}$ being the symbol transmitted from antenna $i\left(1 \leq i \leq n_{t}\right)$ at time $k\left(1 \leq k \leq N_{l}\right)$. Due to the special structure of the S-QSTTC, the transmitted codeword matrix can be written as $\mathbf{D}=\left(\mathbf{C}_{1} \mathbf{C}_{2} \cdots \mathbf{C}_{N_{l} / n_{t}}\right)^{T}$ with appropriately arranged constituent $n_{t} \times n_{t}$ STBCs selected from a certain super quasi-orthogonal code set. $\mathbf{h}$ is a $\left(n_{t} \times 1\right)$ complex vector with $h_{i}$ describing the complex valued channel gain between transmit antenna $i$ and the receive antenna. The channel is assumed to be constant during each frame length $N_{l}$ and changes from one frame to the another. $\mathbf{n}$ is the additive, white complex valued Gaussian noise (AWGN) with variance $\sigma_{n}^{2}$. Note that we assume the frame length $N_{l}$ as a multiple of $n_{t}$.

\section{CODE PERFORMANCE}

The data transmission at each time index $\mathrm{t}$ corresponding to a specific trellis branch can be described by

$$
\mathbf{y}_{t}=\sqrt{E_{b}} \mathbf{C}_{t}^{\Theta_{1}, \Theta_{2}} \mathbf{h}+\mathbf{n} .
$$

This equation can be rewritten by complex conjugation of the second and third element of $\mathbf{y}_{t}$ if $\mathbf{C}_{t}^{\Theta 1, \Theta 2}$ is taken from (5), or by complex conjugation of the second and the fourth element of $\mathbf{y}_{t}$, if $\mathbf{C}_{t}^{\Theta_{1}, \Theta_{2}}$ is taken from (6), as

$$
\tilde{\mathbf{y}}_{t}=\mathbf{H}_{t, v}^{\Theta_{1}, \Theta_{2}} \mathbf{s}+\tilde{\mathbf{n}},
$$

where $\mathbf{H}_{t, v}^{\Theta_{1}, \Theta_{2}}$ is an equivalent, virtual $(4 \times 4)$ channel matrix consisting of elements obtained from the $(4 \times 1)$ channel vector $\mathbf{h}$, including some sign inversions and complex conjugate versions of the original channel coefficients $h_{i} . \mathbf{s}$ is the $(4 \times 1)$ vector of transmitted information symbols $s_{1}$ to $s_{4}$. The quasi-orthogonality of the QSTBCs becomes evident, when maximum ratio combining (MRC) of $\tilde{\mathbf{y}}_{t}$ is applied at the receiver, described by

$$
\mathbf{r}_{t}=\left(\mathbf{H}_{t, v}^{\Theta_{1}, \Theta_{2}}\right)^{H} \mathbf{H}_{t, v}^{\Theta_{1}, \Theta_{2}} \mathbf{s}+\left(\mathbf{H}_{t, v}^{\Theta_{1}, \Theta_{2}}\right)^{H} \tilde{\mathbf{n}} .
$$

The resulting $(4 \times 4)$ Grammian channel matrix in case of a constituent EA code $\mathbf{C}_{t}^{\Theta_{1}, \Theta_{2}}$ results in

$$
\mathbf{G}_{t, E A}^{\Theta_{1}, \Theta_{2}}=\left(\mathbf{H}_{t, v}^{\Theta_{1}, \Theta_{2}}\right)^{H} \mathbf{H}_{t, v}^{\Theta_{1}, \Theta_{2}}=\mathrm{h}^{2}\left(\begin{array}{cccc}
1 & 0 & 0 & X_{1} \\
0 & 1 & -X_{1} & 0 \\
0 & -X_{1} & 1 & 0 \\
X_{1} & 0 & 0 & 1
\end{array}\right)
$$

and indicates a partial decoupling of the information symbols after the MRC. $h^{2}=\left|h_{1}\right|^{2}+\left|h_{2}\right|^{2}+\left|h_{3}\right|^{2}+\left|h_{4}\right|^{2}$ characterizes the resulting channel gain, whereas $X_{1}$ is a channel dependent symbol interference parameter, given by

$$
X_{1}=2 \operatorname{Re}\left\{e^{j \Theta_{1}} h_{1} h_{4}^{*}-e^{j \Theta_{2}} h_{2} h_{3}^{*}\right\} / h^{2} .
$$

For a constituent ABBA code $\mathbf{C}_{t}^{\Theta_{1}, \Theta_{2}}$ the resulting $(4 \times 4)$ Grammian channel matrix results in:

$$
\mathbf{G}_{t, A B B A}^{\Theta_{1}, \Theta_{2}}=\left(\mathbf{H}_{t, v}^{\Theta_{1}, \Theta_{2}}\right)^{H} \mathbf{H}_{t, v}^{\left(\Theta_{1}, \Theta_{2}\right)}=\mathrm{h}^{2}\left(\begin{array}{cccc}
1 & 0 & X_{2} & 0 \\
0 & 1 & 0 & X_{2} \\
X_{2} & 0 & 1 & 0 \\
0 & X_{2} & 0 & 1
\end{array}\right)
$$

with $X_{2}$ given as:

$$
X_{2}=2 \operatorname{Re}\left\{e^{j \Theta_{1}} h_{1} h_{3}^{*}+e^{j \Theta_{2}} h_{2} h_{4}^{*}\right\} / h^{2} .
$$

It is well known that $\mathbf{G}_{t}^{\Theta_{1}, \Theta_{2}}$ should approximate a scaled identity-matrix as far as possible to achieve ideal data stream decoupling. Consequently, $|X|$ should be as small as possible. From Eqn. (10) it can be seen that the QSTBC can 
not completely decouple the signal streams like an OSTBC. The interference parameter $X$ deteriorates the performance of the code according to the actual channel parameters. The smaller $|X|$ is the closer is the code to an orthogonal code with $\mathbf{G}=h^{2} \mathbf{I}$ and vanishing symbol interference. In [5], [9] it has been shown that the scaling-factor $h^{2}$ as well as the respective interference parameter $X$ determines the error probability of QSTBC. Normalizing the mean energy of the transmit symbols to 1 , the bit error probability can be approximated as

$$
\mathrm{BER} \approx \operatorname{erfc} \sqrt{\left(h^{2}\left(1-X_{i}^{2}\right) / 4 \sigma_{n}^{2}\right)} .
$$

Since $X$ in (11) and in (13) is always a sum or a difference of two terms which are the product of two channel values, it is clear that the parameters $\Theta_{1}$ and $\Theta_{2}$ influence the value of $X$ too. In spatially uncorrelated channels, this has no effect on the mean performance of the code. However, in [10] it has been shown that QSTBCs with essentially the same code properties but different transmission properties behave quite different in spatially correlated channels due to the different values of the resulting interference parameter $X$.

To model correlated channels the following channel correlation matrix is used:

$$
\mathbf{R}_{h h}=\mathrm{E}\left[\mathbf{h h}^{H}\right]=\left[\begin{array}{cccc}
1 & \rho & \rho^{2} & \rho^{3} \\
\rho & 1 & \rho & \rho^{2} \\
\rho^{2} & \rho & 1 & \rho \\
\rho^{3} & \rho^{2} & \rho & 1
\end{array}\right] .
$$

$\rho$ is assumed here as a real positive correlation value with $0 \leq \rho \leq 1$. To describe the influence of $\Theta_{1}$ and $\Theta_{2}$ on the performance of the resulting code, we look into the mean value $E\left\{\left|X_{i}\right|\right\}$ of the respective interference term $X_{i}$ for various values of the correlation factor $\rho$. In [9] it has been shown that QSTBCs with a small mean value of $E\left\{\left|X_{i}\right|\right\}$ performs better than a QSTBC with a higher value of $E\left\{\left|X_{i}\right|\right\}$. Hence, $E\left\{\left|X_{i}\right|\right\}$ can be used as a quality measure for QSTBCs.

In Tab. 2 we have listed $E\{|X|\}$ for the different constituent codes taken from Tab. 1. These values have been obtained by simulations of correlated channels. For no channel correlation $(\rho=0)$ all constituent codes have the same mean value $E\{|X|\}$. Thus all QSTBCs perform equally well. With increasing channel correlation $(\rho>0)$ EA-codes $(5)$ with $\Theta_{1}=\Theta_{2}=0$ and $\Theta_{1}=\Theta_{2}=\pi$ show a smaller value of $E\{|X|\}$ than the ABBA-codes (6). Therefore the EAtype QSTBCs perform better than the ABBA-type QSTBCs. For $\Theta_{1}=\pi, \Theta_{2}=0$ and $\Theta_{1}=0, \Theta_{2}=\pi$ the ABBA-type QSTBCs have a smaller mean value $E\{|X|\}$ than the EAtype codes. Therefore, with these parameter values of $\Theta_{1}$ and $\Theta_{2}$ the ABBA-type QSTBCs perform better than EAtype codes. Obviously, $\Theta_{1}$ and $\Theta_{2}$ change the value of the interference parameter and thus strongly effect the performance of the constituent QSTBCs and consequently the performance of the S-QSTTCs.

\begin{tabular}{|c|c|c|c|}
\hline \multicolumn{3}{|c|}{$\Theta_{1}=\Theta_{2}=0 ; \Theta_{1}=\Theta_{2}=\pi$} \\
\hline & $\rho=0$ & $\rho=0.75$ & $\rho=0.95$ \\
\hline$E\left\{\left|X_{E A}\right|\right\}$ & 0.375 & 0.32 & 0.18 \\
\hline$E\left\{\left|X_{A B B A}\right|\right\}$ & 0.375 & 0.53 & 0.8 \\
\hline$\Theta_{1}=\pi, \Theta_{2}=0 ; \Theta_{1}=0, \Theta_{2}=\pi$ \\
\hline & $\rho=0$ & $\rho=0.75$ & $\rho=0.95$ \\
\hline$E\left\{\left|X_{E A}\right|\right\}$ & 0.375 & 0.56 & 0.8 \\
\hline$E\left\{\left|X_{A B B A}\right|\right\}$ & 0.375 & 0.28 & 0.16 \\
\hline
\end{tabular}

Table 2: $E\{|X|\}$ of the constituent codes used in Tab. 1 .

\section{SIMULATION RESULTS}

The only constraints for the rotation parameters $\Theta_{1}$ and $\Theta_{2}$ of the constituent QSTBCs proposed in [7] and [8] are such that they must not expand the original signal constellation. But as we have shown above, the rotation parameters essentially influence the interference parameter of the constituent codes and therefore also influence the performance of the resulting S-QSTTCs at least on spatially correlated channels. In the following, we will shown that an appropriate selection of $\Theta_{1}$ and $\Theta_{2}$ for the constituent Q-STBCs is essential for a good error performance of the resulting S-QSTTC in case of spatially correlated channels.

\subsection{S-QSTTC on Correlated MIMO Channels}

We simulated the frame error ratio (FER) of the S-QSTTCs described in Fig. 1 and Tab. 1 as a function of $E_{b} / N_{0}$ on correlated channels with correlation factors $\rho=0$ and $\rho=0.95$. The simulations have been performed using a frame length of $N_{l}=128$ and a maximum likelihood decoder. Four transmit antennas and one receive antenna have been used. The information rate was $2 \mathrm{bit} / \mathrm{channel}$ use using 4QPSK transmit symbols.

In Fig. 2 the simulation results for the S-QSTTCs built with the EA-type and the ABBA-type QSTBCs for Trellis-Code example 1 (Tab. 1) are shown. For the signal transmission from the upper node using the constituent EA-type QSTBC with $\mathbf{C}_{E A}^{I}=\mathbf{C}_{E A}^{0,0}(E\{|X|\}=0.18)$ performs better than the constituent ABBA-type QSTBC with $\mathbf{C}_{A B B A}^{I}=\mathbf{C}_{A B B A}^{0,0}$ $(E\{|X|\}=0.8)$ on correlated channels with $\rho=0.95$ (Tab. 2). For the lower node, the constituent ABBA-type QSTBC with $\mathbf{C}_{A B B A}^{I I}=\mathbf{C}_{A B B A}^{0, \pi}(E\{|X|\}=0.16)$ performs better than the constituent EA-type QSTBC with $\mathbf{C}_{E A}^{I I}=\mathbf{C}_{E A}^{0, \pi}\left(E\left\{\left|X_{E A}\right|\right\}=\right.$ 0.8 ). Since every trellis state (and so every constituent QSTBC) is used with equal probability, the resulting EAtype S-QSTTC shows the same overall error performance as the ABBA-type S-QSTTC on correlated channels.

For the Trellis-Code example 2, both constituent ABBAtype QSTBCs $\left(\mathbf{C}_{A B B A}^{I}=\mathbf{C}_{A B B A}^{\pi, 0}, \mathbf{C}_{A B B A}^{I I}=\mathbf{C}_{A B B A}^{0, \pi}\right)$ with $E\{|X|\}$ $=0.16$ have better performance than the corresponding EAtype QSTBCs $\left(\mathbf{C}_{E A}^{I}=\mathbf{C}_{E A}^{\pi, 0}, \mathbf{C}_{E A}^{I I}=\mathbf{C}_{E A}^{0, \pi}\right)$ with $E\{|X|\}=0.8$ 


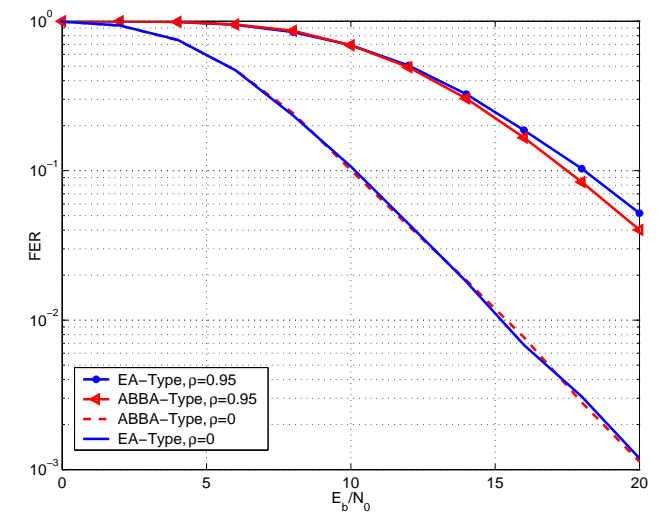

Figure 2: FER of Code 1 on Correlated Channels, $\rho=0$ and 0.95

on correlated channels with $\rho=0.95$. Therefore, the resulting ABBA-type S-QSTTC shows a much better error performance than the EA-type S-QSTTC. The results for TrellisCode 2 are shown in Fig. 3.

Finally, the results for Trellis-Code example 3 (Fig. 4) show

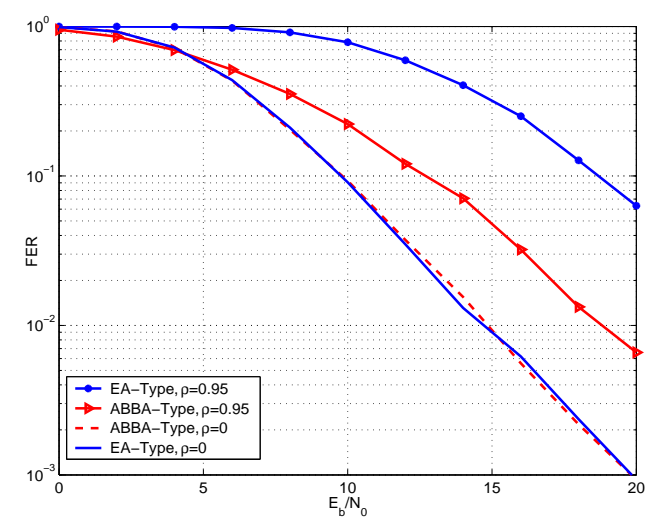

Figure 3: FER of Code 2 on Correlated Channels, $\rho=0$ and 0.95

that the EA-type S-QSTTC performs better than the ABBAtype S-QSTTC, because now both constituent EA-type QSTBCs have smaller mean values of $X$ on correlated channels than the ABBA-type QSTBCs.

As demonstrated in [8] the performance of an S-QSTTC can be improved using full-diversity QSTBCs with multi dimensional signal rotation. However, in this case the transmitter is more complex, because higher order symbol constellations have to be decoded.

\section{CONCLUSION}

In this paper we have analyzed and discussed six super quasiorthogonal space-time trellis codes for four transmit anten-

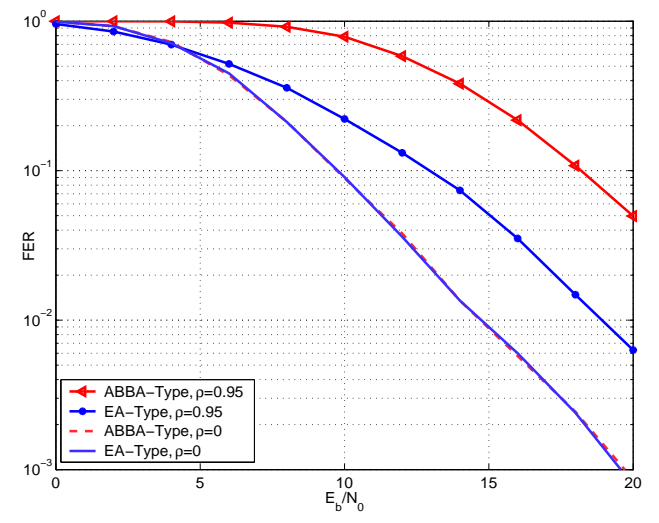

Figure 4: FER of Code 3 on Correlated Channels, $\rho=0$ and 0.95

nas on spatially correlated MIMO channel. It has been shown that EA-type S-QSTTCs and ABBA-type S-QSTTCs perform equally well in spatially uncorrelated MIMO channels. However, on highly correlated channels EA-type codes and ABBA-type codes perform quite different. In order to optimize the error performance we need different labelling of the trellis branches in case of EA-type and ABBA-type constituent Q-STBCs as shown in our simulation results.

\section{REFERENCES}

[1] V. Tarokh, N. Seshadri, A.R. Calderbank, Space-time codes for high data rate wireless communication: Performance analysis and code construction, IEEE Trans. Inform. Theory, vol. 44, pp. 744-765, Mar. 1998.

[2] S.M Alamouti "A simple diversity technique for wireless communications," IEEE J. Selected Areas of Comm., vol. 16, no. 8, pp. 14511458, Oct. 1998

[3] V. Tarokh, H. Jafarkhani and A.R. Calderbank, "Space-time block codes from orthogonal designs," IEEE Trans. Inf. Theory, vol. 45, pp. 1456-1467, July 1999.

[4] O. Tirkkonen, A. Boariu, A. Hottinen, "Minimal nonorthogonality rate one space time block codes for 3+ Tx antennas", in Proc. IEEE Int. Symp. on Spread Spectrum Techniques and Applications 2002, pp. 429-432, Sept. 2000.

[5] C.F. Mecklenbräuker, M. Rupp, "Flexible space-time block codes for trading quality of service against data rate in MIMO UMTS, "EURASIP Journal on Applied Signal Processing, no. 5, pp. 662-675, May 2004.

[6] S. Alamouti, V. Tarokh, P. Poon, "Trellis-coded modulation and transmit diversity: Design criteria and performance evaluation", in Proc. IEEE ICUPC'98, pp. 703-707, Oct. 1998.

[7] H. Jafarkhani and N. Seshadri, Super-orthogonal space-time trellis codes, IEEE Trans. Inform. Theory, vol. 49, pp. 937-950, Apr. 2003.

[8] H. Jafarkhani, N. Hassanpour, "Super-quasi-orthogonal space-time trellis codes for four transmit antennas", IEEE Trans. on Wireless Comm., vol. 4., pp. 215- 227, Jan. 2005.

[9] B. Badic, H. Weinrichter, M. Rupp, "Comparison of non-orthogonal space-time block codes using partial feedback in correlated channels", in Proc. IEEE SPAWC 2004, Lisboa, Portugal, July 2004.

[10] B. Badic, M. Rupp, H. Weinrichter, "Quasi-Orthogonal Space-Time Block Codes: Approaching Optimality", accepted for EUSIPCO'05, Antalya, Turkey Sept. 2005 . 